\title{
Mise à jour de la distribution de l'abeille du lierre, Colletes hederae SCHMIDT \& WESTRICH (Hymenoptera, Colletidae) en Europe
}

\author{
Par Nicolas J Vereecken ${ }^{\star}$, Hans Schwenninger ${ }^{\star \star}$, Andrej GogalA ${ }^{\star \star \star}$, et Stuart PM RobertS ${ }^{\star \star \star \star}$
}

\section{Ecologie de 1'abeille du lierre}

L'abeille du lierre, Colletes hederae SCHMIDT \& WESTRICH est une espèce décrite il y a un peu plus de 15 ans seulement sur base de spécimens récoltés en Allemagne et en Croatie. Son étroite ressemblance à $C$. succinctus lui avait d'abord valu le statut de sous-espèce, jusqu'à ce que certains caractères morphologiques diagnostiques d'une part, et son écologie d'autre part lui valurent d'être élevée au statut spécifique. Son épithète spécifique est lié à sa plante hôte de prédilection, Hedera helix (le lierre, Araliaceae) (Schmidt \& Westrich 1993) (Figure 1). Les sites de nidification prennent parfois l'allure de véritables agrégations comptabilisant plusieurs dizaines de nids au mètre carré (Figure 2).

Depuis sa description, cette espèce a fait l'objet de plusieurs études visant à préciser les limites de sa distribution en Europe occidentale, ainsi que la nature de son régime alimentaire. Ainsi, il est rapidement apparu que l'abeille du lierre jouit d'une large distribution en Europe comprenant en outre l'Angleterre, la Belgique, l'Espagne, la France, la Grèce, l'Italie, le Luxembourg, les Pays-Bas, la Slovénie et la Suisse (voir Vereecken et al. 2006). Les récentes études palynologiques de Müller \& Kuhlmann (2008) et Westrich (2008) basées sur les masses de pollen récoltées par les femelles et sur les pains de pollen accumulés dans les cellules larvaires (Figure 3) ont mis en évidence que l'espèce n'est pas strictement monolectique (voir Schmidt \& Westrich 1993; Bischoff et al. 2005) mais qu'elle récolte occasionnellement du pollen sur d'autres plantes parmi lesquelles des Odontites (Scrophulariaceae), des Calluna (Ericaceae) et autres Cichorioidae (Müller \& Kuhlmann 2008).

\section{Projet sur l'abeille du lierre}

Depuis la dernière mise à jour des observations relatives à l'abeille du lierre, un réseau d'observateurs s'est mis en place dans divers pays d'Europe

* Evolution Biologique \& Ecologie, Université Libre de Bruxelles CP 160/12, Av. F.D. Roosevelt 50, B-1050 Bruxelles, Belgique.

E-mail: nicolas.vereecken@ulb.ac.be

** Büro Entomologie + Ökologie Goslarer Str. 53 D-70499

Stuttgart, Deutschland. E-mail: H.u.K.Schwenninger@t-online.de *** Slovenian Museum of Natural History, Preøernova 20, SI-1001 Ljubljana, Slovenia. E-mail: andrej.gogala@guest.arnes.si ***** Centre for Agri-Environmental Research (CAER), School of Agriculture, Policy and Development, University of Reading, RG6 6AR, UK. E-mail: eucera@yahoo.com

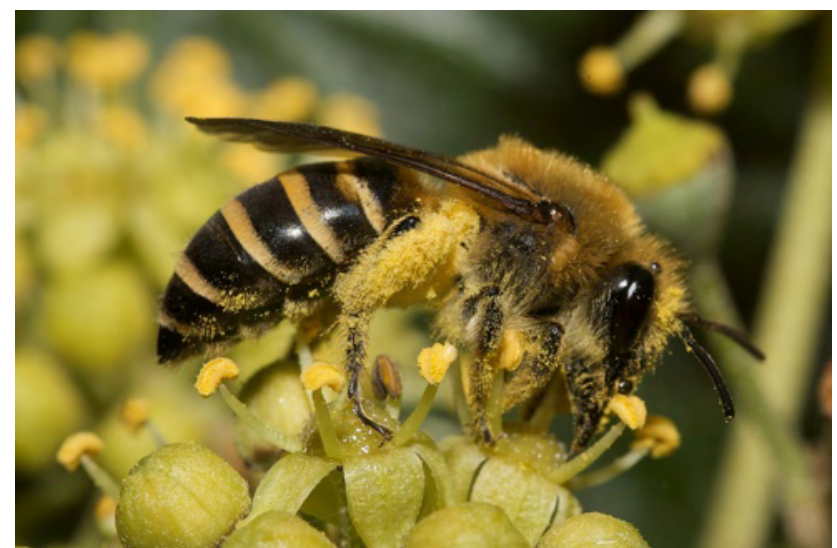

Figure 1. Femelle de Colletes hederae SCHMIDT \& WESTRICH chargée de pollen de lierre sur ses pattes postérieures (Photo NJ Vereecken)

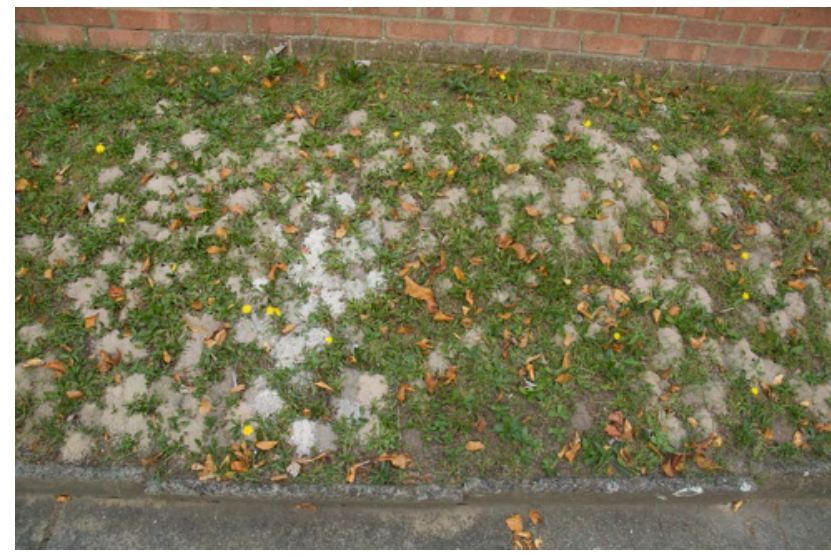

Figure 2. Agrégation de Colletes hederae SCHMIDT \& WESTRICH installée dans un jardin (Photo NJ Vereecken)

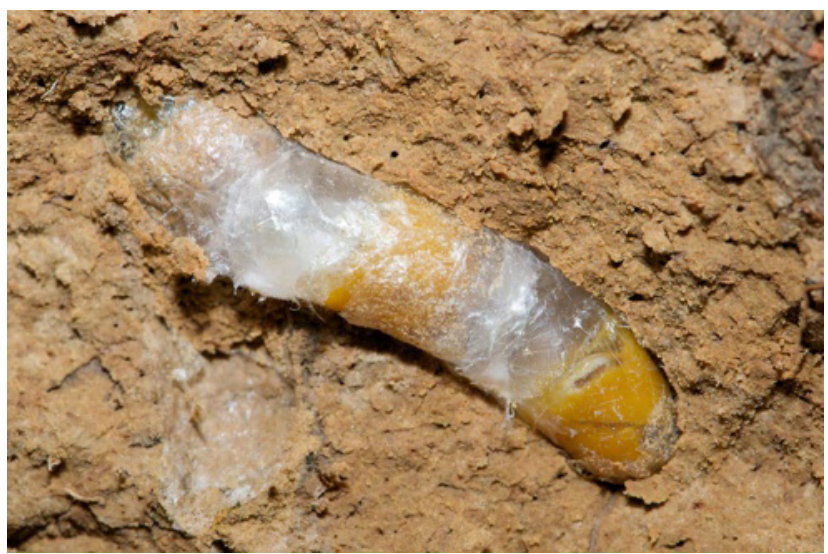

Figure 3. Cellules larvaires de Colletes hederae SCHMIDT \& WESTRICH. Le pollen de lierre est visible au travers de la paroi (Photo NJ Vereecken) 


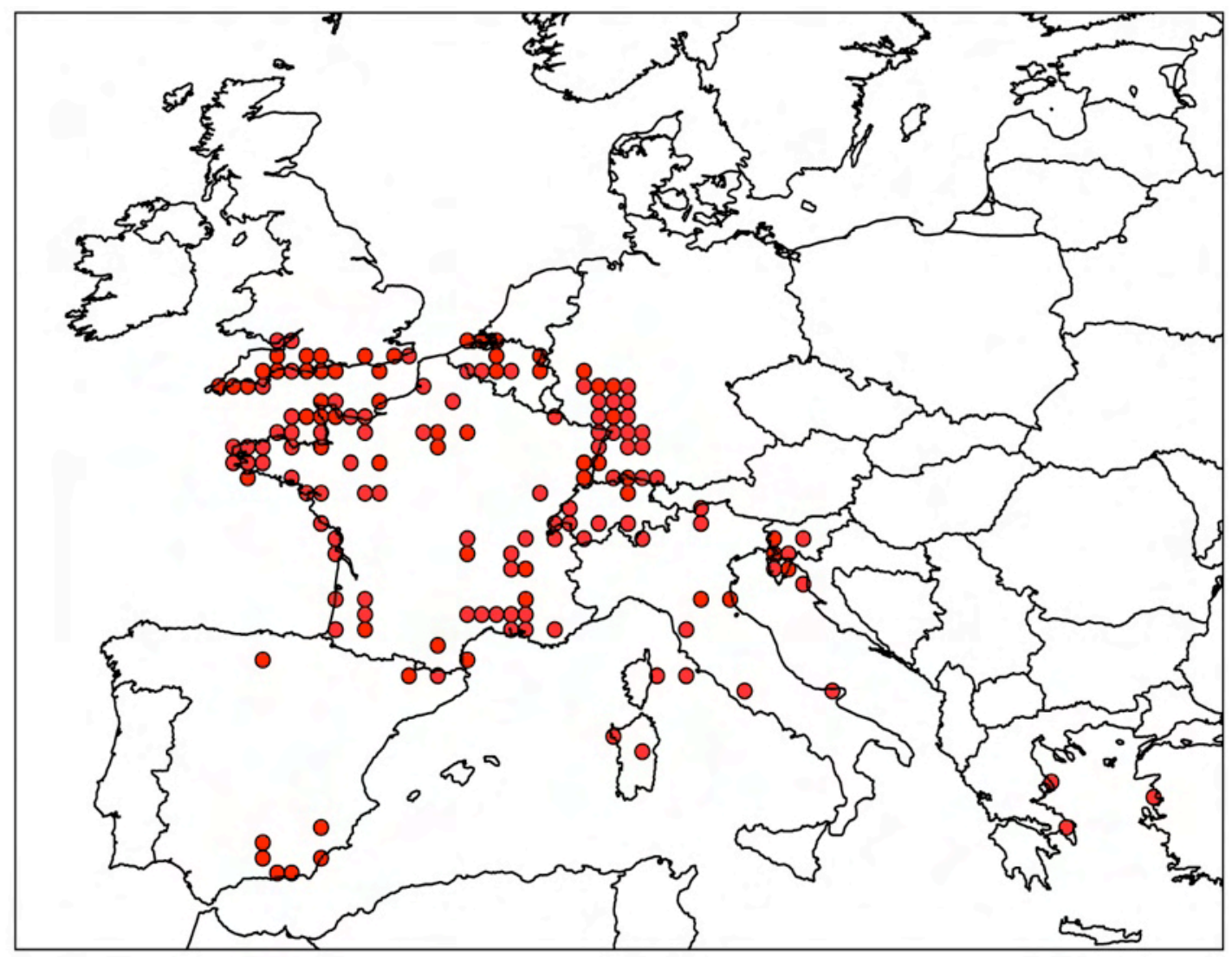

Figure 4. Mise à jour de la distribution des observations de Colletes hederae SCHMIDT \& WESTRICH en Europe.

occidentale, avec pour objectif de mettre à jour la carte des distributions relatives à l'abeille du lierre. Nous présentons à la Figure 4 la dernière version de cette carte qui reprend toutes les données auxquelles nous avons pu avoir accès jusqu'au début de l'hiver 2008. Plusieurs aspects de cette figure nous semblent remarquables :

- des données récoltées par Ortiz-Sanchez \& Castro (2008) ont permis de repousser les limites de la distribution de cette espèce jusqu'à l'extrême sud de la Péninsule Ibérique;

- la France est désormais bien mieux couverte qu'il y a 2-3 années mais peu de données sont disponibles pour l'intérieur du pays, tout comme en Espagne, où l'espèce est plus que probablement présente;

- l'espèce semble rencontrer une limite septentrionale au-delà de la frontière belgonéérlandaise et au-delà de la ligne rejoignant l'estuaire de Bristol et celui de la Tamise (river Thames) en Angleterre.

\section{Perspectives}

Il reste maintenant à examiner l'expansion de l'abeille du lierre vers l'est en Europe continentale (sud de l'Allemagne (voir Frommer 2008), Autriche, etc., ainsi que la colonisation de nouveaux habitats au nord de la limite actuelle de sa distribution en Angleterre et en Europe occidentale. Nous encourageons tous les naturalistes à se pencher sur cette espèce qui promet d'intéressantes perspectives d'observations au cours des prochaines saisons, à commencer par celle qui débutera dès fin août 2009.

\section{Remerciements}

Nous remercions Rosita Moenen (Bennekom, NL), Kobe Janssens, Jan Smit (Duiven, NL) et Javier Ortiz (Almeria, ES) et les membres des réseaux BWARS, Database European Invertebrate Survey - the Netherlands, Wildbienen-Kataster, Arbeitsgemeinschaft Hessischer Hymenopterologen et Hautflügler-Kataster Rheinland-Pfalzpour nous avoir fourni l'accès à leurs données biogéographiques.

\section{Références bibliographiques}

Bischoff I, Eckelt E \& Kuhlmann M, 2005. On the Biology of the Ivy-Bee Colletes hederae SCHMIDT \& WESTRICH, 1993 (Hymenoptera, Apidae). Bonner zoologische Beiträge 53 (1/2): 27-36.

Frommer U, 2008. Grundlagen der Ausbreitung und aktuellen nördlichen Verbreitung der Efeu-Seidenbiene Colletes hederae SCHMIDT \& WESTRICH, 1993 in Deutschland. Mitt. internat. entomol. Ver. $33(1 / 2): 59-74$.

Moenen R 2005. Waarnemingen aan de klimopbij (Hymenoptera: Apidae). Entomologische Berichten 65: 145-148.

Müller A \& Kuhlmann M, 2008. Pollen hosts of western palaearctic bees of the genus Colletes (Hymenoptera: Colletidae): the Asteraceae paradox. Biological Journal of the Linnean Society 95: 719-733.

Ortiz-Sanchez J \& Castro L, 2008. No es Colletes hederae SCHMIDT \& WeSTRICH, 1993 todo lo que a la flor de la hiedra acude (Hymenoptera, Apoidea, Colletidae). Boletín Sociedad Entomológica Aragonesa 42: 337-339

Schmidt K \& Westrich P, 1993. Colletes hederae n. sp., eine bisher unerkannte, auf Efeu (Hedera) spezialisierte Bienenart (Hymenoptera: Apoidea). Entomologische Zeitschrift 103 (6): 89-112.

Vereecken N, Toffin E \& Michez D, 2006. Observations relatives à la biologie et à la nidification d'abeilles psammophiles d'intérêt en Wallonie. 2. Observations estivales et automnales. Parcs et Réserves 61(4): 12-20.

Westrich P, 2008. Flexibles Pollensammelverhalten der ansonsten streng oligolektischen Seidenbiene Colletes hederae SCHMIDT \& WESTRICH (Hymenoptera: Apidae). Eucera 2: 17-29. 